\title{
Report from Strasbourg
}

\author{
MRS-Europe Spring Meeting
}

The 1985 Spring Meeting of MRS-Europe which was held at the Council of Europe in Strasbourg, France, May 13-15, demonstrated the growing cooperation in and enthusiasm for the MRS-style research forum. More than 130 papers, posters presentations, plenary lectures, and roundtable issues were featured at the meeting. Approximately 300 scientists from Europe, the United States, Japan and elsewhere participated in the three symposia which focused on topics ranging from developments in the structure, properties, and processing of electronic materials to processing and characterization of materials under microgravity, low temperatures, and high pressure.

Three plenary lectures kicked off the meeting on Monday by providing state-ofthe-art reviews of important scientific developments on the three general areas emphasized in the symposia. Speakers and their topics were : A. Y. Cho (AT\&T Bell Laboratories), "Recent Developments in III-V Compound Semiconductor Materials and Devices Grown by MBE"; W. M. Lovell (Marconi Company Ltd.), System Relevance of SOI Technologies"; and D. T. J. Hurle (RSRE), "Influence of Gravity on Crystal Growth." (See a summary of Dr. Hurle's presentation elsewhere in this article.)

The roundtable session on industry-university collaboration from the industry viewpoint featured five distinguished panelists: W. Freiesleben (Wacker Chemitronic), C. Hilsum (GEC Research Laboratories), P. Leclerc (Thomson-CSF), F. Meijer (Philips Research Laboratories), and C. Weyrich (Siemens Corporate Research and Development). The panel discussed the present trends and potential activity of joint industry-university research programs, specifically reviewing industry limitations and requirements pertaining to such ventures, and examining various types of cooperative $R \& D$ programs (e.g., personnel exchanges, contracts, and technology transfer centers).

The relaxing atmosphere of the spacious Council of Europe, combined with the mild spring weather and exquisite local Alsatian cuisine made the week enjoyable as well as intellectually stimulating. The logistical management of the meeting, under the direction of MRS-Europe's P. Siffert and the staff of CRN, was expertly handled, adding another pleasant dimension to the experience.

The scientific presentations in all the sessions were augmented by frequent exchange between speaker and audience. In fact, one can speculate about the impression made on the various general public tour groups, which had the opportunity to observe from the gallery the main auditorium (which is normally the venue of international political forums) the proceedings of some lively portions of Symposium I on "Energy Beam-Solid Interactions and Transient Thermal Processing."

The following summaries provided by the symposia chairmen highlight some of the interesting developments presented during the meeting. Symposium chairmen were: V. T. Nguyen (CNET) and A. G. Cullis (RSRE), Symposium I: "Energy Beam-Solid Interactions and Transient Thermal Processing"; H Ahlborn (Universität Hamburg), H. Fredriksson (Royal Institute of Technology, Stockholm), and E. Luscher (Technische Universität, Garching), Symposium II: "Materials Under Extreme Conditions"; and K. Ploog (Max Planck Institute) and N. T. Linh (ThomsonCSF), Symposium III: "Semiconductor Quantum Well Structures and Superlattices."

Proceedings of the meeting will be published by Les Editions de Physique later this year, and will also be available for purchase through MRS. See further information on the proceedings in upcoming issues of MRS BULLETIN.

\section{Energy Beam-Solid Interactions and Transient Thermal Processing \\ (Symposium I)}

This symposium chaired by V. T. Nguyen (CNET, France) and A. G. Cullis (RSRE, UK), covered a wide range of topics from the fundamentals of ultra-fast beam-solid interactions and ion and electron beam induced materials modifications to laser chemical and transient thermal processing and the recrystallization of silicon-on-insulator (SOI). An introduction to the symposium was provided by $W$. M. Lovell (Marconi Company) with a plenary talk on the systems relevance of SOI technologies. The general sessions included 76 papers in both oral or poster formats.

The opening sessions of the symposium concentrated on the response of semiconductors to high-intensity laser pulses. Four invited talks were given. W. L. Brown (AT\&T Bell Laboratories) reviewed dynamic studies of high-speed crystal growth; A. G. Cullis (RSRE) described the fundamental characteristics of fast crystal growth processes; S. U. Campisano (Catania University) outlined the effects of impurities on amorphous phase quenching; and D. Hulin (ENS) detailed the evolution of silicon irradiated with femtosecond pulses. Further consideration of dense electron-hole plasma behavior was given in contributed papers. Brown reports that, despite the many fast transient measurements, the quantitative aspects of superheating and supercooling with fast melting and solidification are still uncertain and require careful computer modeling in conjunction with the experimental measurements. Evidence for the explosive crystallization of amorphous silicon in to fine grain polycrystalline material was discussed. Questions concerning the transition from dense electron-hole plasma to molten material on timescales of a picosecond or less are largely unanswered though they are receiving continued vigorous theoretical and experimental

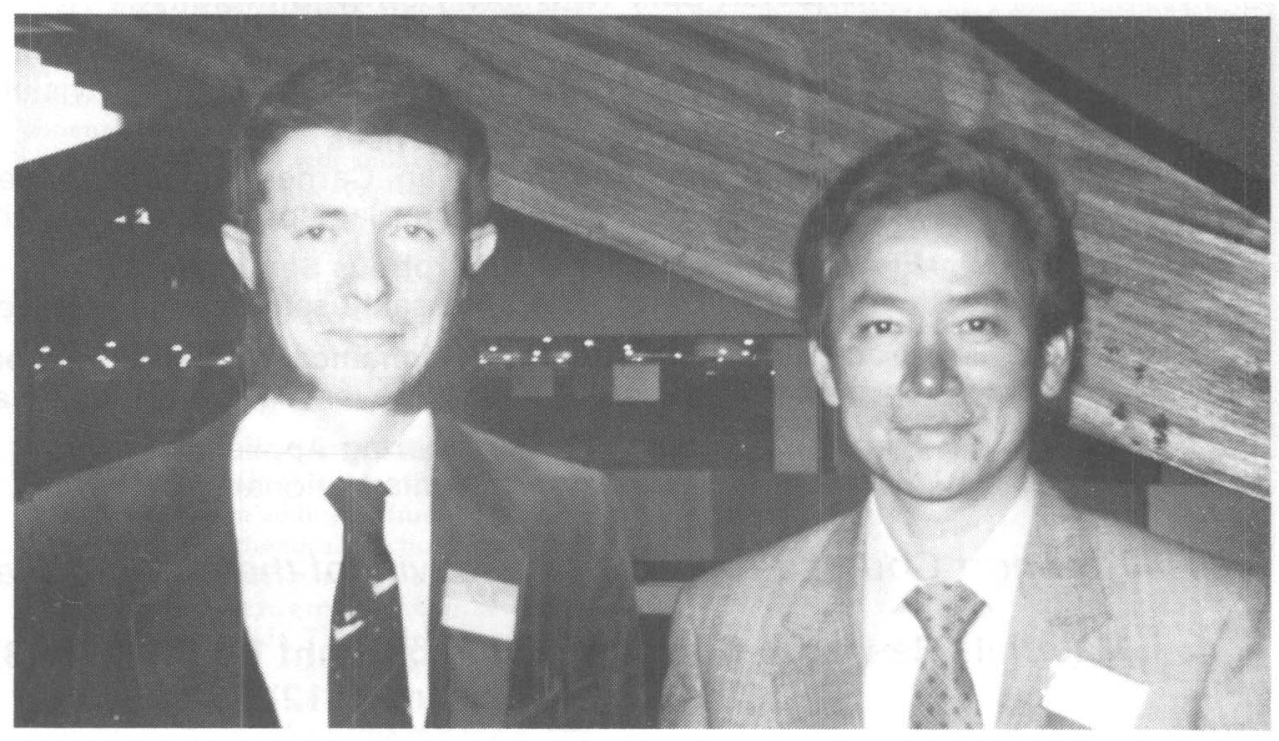

A. G. Cullis (left) and V. T. Nguyen, chairmen of Symposium I. 
attention

The sessions covering laser chemical processing were also introduced by four invited papers. D. Bäuerle Johannes Kepler University) gave a general review of the topic, while D. J. Ehrlich (Lincoln Laboratories) focused on laser direct patterning. J. Siejka (ENS) described UV photon-beam-oxide interactions, and E. Fogarassy (CRNPHASE) treated semiconductor processing with excimer lasers. Bäuerle noted that the contributed papers mostly concentrated on LCVD, with special attention being paid to the careful characterization of the deposits. A growing interest in studying photolytic and pyrolytic decomposition for each investigated system was evident, and by combining these processes, some workers succeeded in improving the quality of deposited layers.

The next session highlighted the use of methods other than laser or rapid thermal annealing for materials modification. The invited presentation by F. W. Saris (FOM) on metastable alloy formation by ion beam mixing addressed a number of key questions, including the advantages of ion mixing over other fabrication techniques (such as ion implantation with annealing or direct coevaporation) and the stability of ionmixed metas table phases. Saris pointed out that some of these issues were also considered in the contributed papers, which included studies of ion mixed metal alloys. Self-annealing during high current density implants of phosphorous into silicon was described, in addition to high-voltage electron beam activated epitaxy and pulsed electron beam annealing investigations.

Two sessions focused on transient materials processing with three invited talks. J. Götzlich (Fraunhofer Institute) outlined the practical aspects of laser and rapid thermal annealing while B. J. Sealy (Surrey University) concentrated on the latter technique as applied to compound semiconductors. $M$. von Allmen described a range of metastable materials formed by pulsed laser irradiation. Some of the contributed papers presented work on materials modification by laser annealing, although most reported studies of rapid thermal annealing. Sealy emphasized the importance of the lat ter technique for advanced semiconductor device processing. The high dopant activations achieved and the control which is obtained over dopant profiles render the multisecond annealing approach most attractive for technological applications.

The final two sessions of the symposium directed attention to the important area of the formation of silicon-on-insulator (SOI) and seven invited papers provided in-depth coverage of critical areas. T. Kamins (HewlettPackard Laboratories) gave an overview of the promises and pitfalls of laser-recrystallized SOI devices and observed that unseed-

"Also see report on developments in three-dimensional integrated circuits on page 5. ed films recrystallized by $C W$-laser radiation are ready for commercial implementation, while grain-boundary-free films required for high-performance applications need further development. T. Nishimura (LSI R\&D Lab, Mitsubishi Electric Corp.) presented impressive examples of the use of the $\mathrm{CW}$ laser approach for the fabrication of multilevel, three dimensional integrated circuits. ${ }^{\star \star}$

$M$. Haond (CNET) reviewed recent advances in light-beam induced recrystallization of SOI films, with the latest results from the use of a lamp heating system, and D. J. Godfrey (GEC Hirst Research Centre) described the use of a dual electron beam system for the recrystallization process. Solid-phase SOI production techniques were also feat ured and L. Jastrzebski (RCA Laboratories) reviewed a CVD epitaxial lateral overgrowth method and pointed to promising reports of lateral to vertical growth ratios significantly greater than unity. G. Bomchil (CNET) discussed the formation and oxidation of porous silicon, and P. L. F. Hemment (Surrey University) gave details of SOI structures produced by high dose oxygen or nitrogen ion implantation. Further work on the latter implantation technique was presented in later contributed papers.

The symposium extended over three days and the use of parallel sessions was avoided for most of the oral presentations. A substantial number of papers were included in a poster session, and this stimulated a large amount of scientific discussion.

\section{Materials Under Extreme Conditions (Symposium II)}

This symposium was devoted to the properties as well as to the production of materials under extreme conditions such as microgravitation, high pressure and low temperature. The field of materials produced under microgravity dealt with fluid physics phenomena, crystal growth, and metallurgy. In the field of crystal growth, D. T. J. Hurle (RSRE, UK) gave an overview of the problems due to natural convection during crystal growth (see summary of his lecture in the box).C. Potard (CEN, France) showed the effect of convection under the influence of gravity on crystals in solutions. P. G. Barber (Longwood College, USA) discussed the problems of growing crystals when the solubility and the temperature density dependence are opposite. In the two other fields, high pressure and low temperature, discussions on Marangoni convection, stability of liquid bridges, and dendritic growth were provided.

See Plenary Lecture Summary on page 10.

\section{Semiconductor Quantum Well Structures and Superlattices \\ (Symposium III)}

This symposium comprised a plenary lecture given by A. Y. Cho (AT\&T Bell Laboratories), 10 invited papers presented by distinguished scientists from Europe Japan and the United States, and 28 conributed papers over the three-day meeting. The nine sessions were attended by more than 100 scientists and engineers, mostly from European countries. The poster session complete with refreshments provided ample opportunity for technical discussion and less-than-serious crosstalks.

The rapid development in the thin-film growth techniques of molecular beam epitaxy (MBE), metalorganic vapor phase epitaxy (MOVPE), and hot wall epitaxy (HWE) was demonstrated by the recent achievements of highly refined ultrathin multilayer structures which exhibit novel physical properties important for new concepts of semiconductor devices. In addition to the well-established quantum well heterostructures made from the III- $V$ semiconductors GaAs/AlAs, GaxIn 1-x As/InP, GaxIn 1-x As/

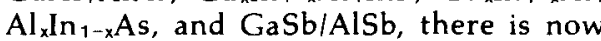
increasing interest in artificial lattices made

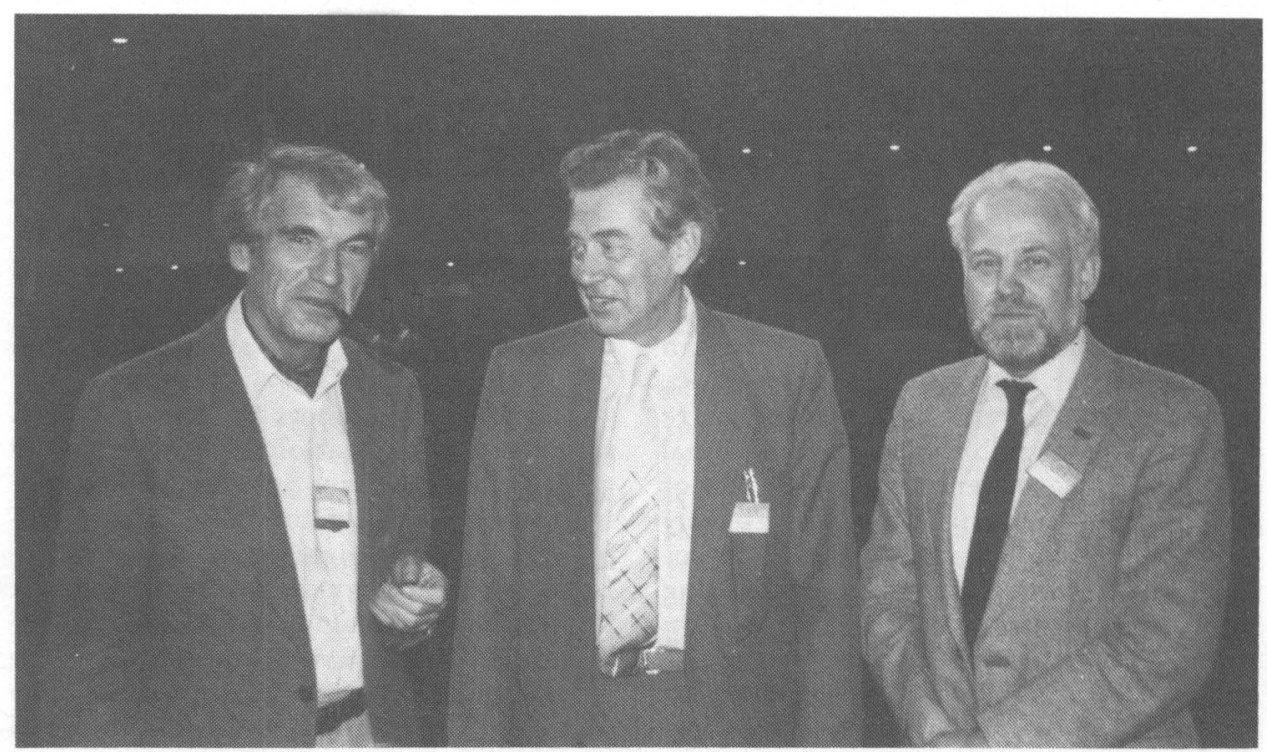

Left to right: E. Lusher, H. Ahlborn, and H. Fredriksson, chairmen of Symposium II. 
of the II-VI semiconductors $\mathrm{CdTe} / \mathrm{HgTe}$, of the IV-VI semiconductors $\mathrm{PbTe} / \mathrm{Sn}_{x} \mathrm{~Pb}_{1-x}$ $\mathrm{Te}$, and of the group-IV-elements $\mathrm{Si} / \mathrm{Ge}$.

Strained-layer superlattices, where the lattice mismatch of the two constituent components is accommodated by elastic strain, now play an important role in extending the concept of "bandgap engineering" in semiconductors. The built-in strain in selectively doped $\mathrm{Si} / \mathrm{Si}_{x} \mathrm{Ge}_{1-x}$ superlattices lead to a confinement of highmobility electrons in the constituent $\mathrm{Si}$ layers. Alternating monolayers of
GaAs/InAs exhibit totally new electronic phenomena.

The large variety of ultrathin multilayer structures requires highly improved assessment techniques. Of particular importance is the microscopic structure at the interface of the constituent layers. It was demonstrated that refined double crystal x-ray diffraction and cross-sectional lattice imaging by transmission electron microscopy (TEM) allow identification of the position of the heterointerface on an atomic scale.

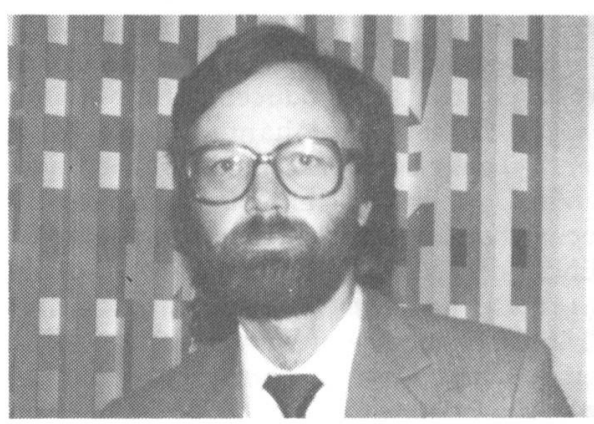

K. Ploog, chairman of Symposium III.

PLENARY LECTURE SUMMARY

\section{Influence of Gravity on Crystal Growth}

\section{T. J. Hurle \\ RSRE}

The principal effects which gravity has on the growth of crystals occurs through buoyancy-driven convection. An additional, but less significant, effect is the phase separation induced by gravity, namely the degassing of a melt and the sedimentation of particulate matter. Convection in principle is beneficial to crystal grow th because it reduces the diffusional barrier to the growth. This is true in practice provided that the flow is stationary. However, in reality on earth convection is frequently turbulent, which leads to unwanted compositional inhomogeneities known as solute striations.

The impetus behind experiments to grow crystals in space is the hope that such crystals will be less defective and more homogeneous because of the dramatic reduction in the buoyancy flow. However, Marangoni (surface tension driven) convection, which is time dependent, can also occur. Therefore, the perceived advantages of the microgravity environment also contain some disadvantages:

- solute striations can be avoided but timedependent Marangoni flow may occur;

- the possibility exists for carrying out basic research into effects which are obscured by buoyancy-driven convection on earth but a residual level also exists which is superimposed by poorly defined " $\mathrm{g}$-jitter" level; and

- very high purity crystals might be obtained by extended use of containerless growth.

Because it is necessary to remove from the vicinity of the crystallizing interface the generated latent heat, temperature gradients develop which give rise to thermal convection. Moreover, except in the case of an elemental material or a congruently melting compound, one or more components will be segregated at the crystallizing interface resulting in a concentration boundary layer in the melt adjacent to the interface. If the melt density is compositiondependent, this too will give rise to convec- tive motion (solutal convection). Crystal growers therefore need to study the convective behavior of their melts and solutions to establish the nature of the flows and to seek to obtain conditions - by varying the experimental parameters or by imposing additional external fields - under which the flow is stationary. Where this cannot be achieved it is important to understand how the growing crystal responds to the timevarying field.

Crystal growers face the problem that, even if the configuration of their apparatus maintain a steady convective flow at the outset of growth, the convection conditions change because convections change as crystal grows (e.g., change melt volume and aspect ratio). Bifurcations to new convective states can occur, and while these new states may also be stationary, the process of transition can violently upset growth conditions. This is most apparent in the Czochralski growth of some oxide crystals where a catastrophic melt flow transition results when the crystal rotation rate and/or the crystal diameter is increased.

On earth the driving force for convective motion is usually so strong that the flow is turbulent. This turbulence both modulates the growth rate of the crystal by modulating the temperature field and the thickness, and hence the admittance, of the mass transport boundary layer because its outer edge is subject to a time variation in the flow. Both of these effects produce a banded distribution of the solute, the form of which depends on the response of the crystal-melt interface.

There are two aspect to consider. First, there are many situations in which the time periodic flow is essentially uncoupled, the melt itself merely acting as a forcing frequency. Second, through its ability to deform, the interface must be considered part of the dynamic system giving rise to morphological-convective instabilities. If the amplitude of the temperature oscillation is sufficiently large, the already grown crystal will melt back during part of the oscillation cycle. Such behavior is often seen, for example, in the pattern of striations in large single crystals of oxides and silicons. In fact, the crystal acts as a very selective filter, having a damped resonant response due to coupling to the characteristic response frequencies of the thermal and solutal boundary layers.

In the spacecraft environment, we may think in terms of externally imposed accelerations rather than of turbulent convection. At high frequencies the whole melt will respond inertially, while at low frequencies the applied oscillatory force will be dissipated by viscous friction generating shear within the liquid. It is this situation, where the shear modulates solute and thermal boundary layers, which is the most deletereous to the homogeneity of the crystal. A power spectrum of maximum allowable acceleration can therefore be defined. This reveals that exceedingly low acceleration levels, down to very low frequencies, are required for truly homogeneous growth in space.

To avoid time periodicity under terres trial conditions, additional external fields such as magnetic field or rotation of the system are necessary. Once the buoyancydriven flow has been reduced until it becomes comparable in energy to that associated with the capillary forces (as can occur in the space environment), coupling between the convective and morphological modes of the system becomes possible. It is anticipated that the forthcoming space experiments will reveal an intriguing new spectrum of behavior.

Recent space experiments on the growth of protein crystals and insoluble phosphates have shown that the technique of counter-diffusing two solutes into a third solution in order to achieve a controlled supersaturation can yield much larger and more perfect crystals than can be obtained on earth. 